\title{
Editorial
}

\section{Nonlinear Analysis and Geometric Function Theory}

\author{
Ljubomir B. Ćirić, ${ }^{1}$ Samuel Krushkal, ${ }^{2}$ Qamrul Hasan Ansari, ${ }^{3}$ \\ David Kalaj, ${ }^{4}$ and Vesna Manojlovic ${ }^{5}$ \\ ${ }^{1}$ Faculty of Mechanical Engineering, University of Belgrade, Belgrade, Serbia \\ ${ }^{2}$ Department of Mathematics, Bar-Ilan University, 5290002 Ramat-Gan, Israel \\ ${ }^{3}$ Department of Mathematics, Aligarh Muslim University, Aligarh 202 002, India \\ ${ }^{4}$ Faculty of Natural Sciences and Mathematics, University of Montenegro, 81000 Podgorica, Montenegro \\ ${ }^{5}$ Mathematical Institute SANU and Faculty of Organizational Sciences, University of Belgrade, 11000 Belgrade, Serbia
}

Correspondence should be addressed to Ljubomir B. Ćirić; lciric@rcub.bg.ac.rs

Received 30 September 2014; Accepted 30 September 2014; Published 10 November 2014

Copyright (c) 2014 Ljubomir B. Ćirić et al. This is an open access article distributed under the Creative Commons Attribution License, which permits unrestricted use, distribution, and reproduction in any medium, provided the original work is properly cited.

Sobolev mappings, quasiconformal mappings, or deformations, between subsets of Euclidean space or manifolds and harmonic maps between manifolds, may arise as the solutions to certain optimization problems in the calculus of variations, as stationary points which are the solutions to differential equations (particularly in conformal geometry). The most recent developments in the theory of planar and space quasiconformal mappings are also related to the theory to degenerate elliptic equations, which include p-Laplace equation whose solutions are $\mathrm{p}$-harmonic maps. In particular, Euclidean harmonic (2-harmonic) maps, which are critical points of Dirichlet's integral, are related to the theory of minimal surfaces and are the primary object of the study in classical potential theory. The complex gradient of the pharmonic operator is quasiregular and it is general feature of nonlinear PDEs.

Harmonic maps have also played a role in compactifications and parametrizations of Teichmuller space, via classical results of Mike Wolf, who gave a compactification in terms of hyperbolic harmonic maps. Teichmuller spaces are central objects in geometry and complex analysis today, with deep connections to quasiconformal mappings (in particular, to extremal problems of quasiconformal mappings), string theory, and hyperbolic 3 manifolds. The study of connection between extremal mappings of finite distortion and harmonic mappings is also initiated. Since its introduction in the early 1980s, quasiconformal surgery has become a major tool in the development of the theory of holomorphic dynamics.
In connection with the general trend of the geometric function theory in Euclidean space to generalize certain aspects of the analytic functions of one complex variable, there is another development related to maps of quasiconformal type, which are solutions of nonlinear secondorder elliptic equations or satisfy certain inequality related to Laplacian and gradient.

Variational analysis, fixed point theory, split feasibility problems, and so forth are also some important and vital areas in nonlinear analysis. During the last two decades, several solution methods for these problems have been proposed and analyzed. These areas include application in optimization, medical sciences, image reconstruction, social sciences, engineering, management, and so forth.

In this special issue, thirteen articles were being published. All these articles have given important contributions to different parts of the above-discussed areas. Among them stands out the very important work of Professor M. Mateljević, one of the most prominent scientists in the geometric function theory. In fact, this issue is devoted to his 65th birthday, as many authors emphasized in their articles.

Ljubomir B. Ćirić Samuel Krushkal Qamrul Hasan Ansari David Kalaj

Vesna Manojlović 


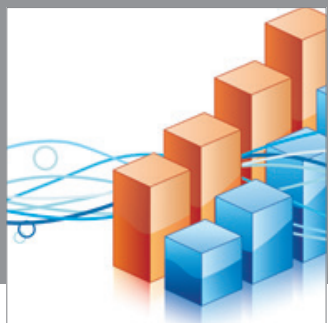

Advances in

Operations Research

mansans

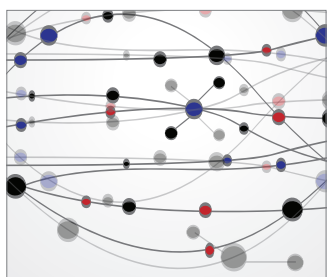

The Scientific World Journal
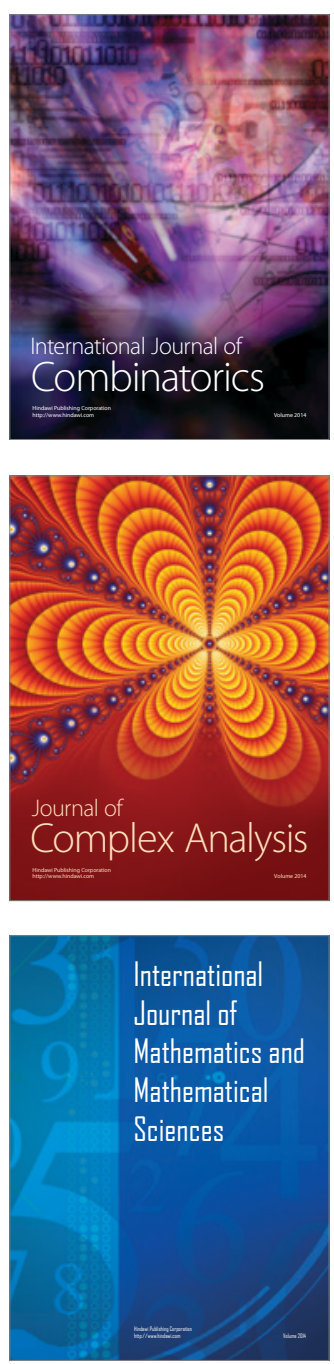
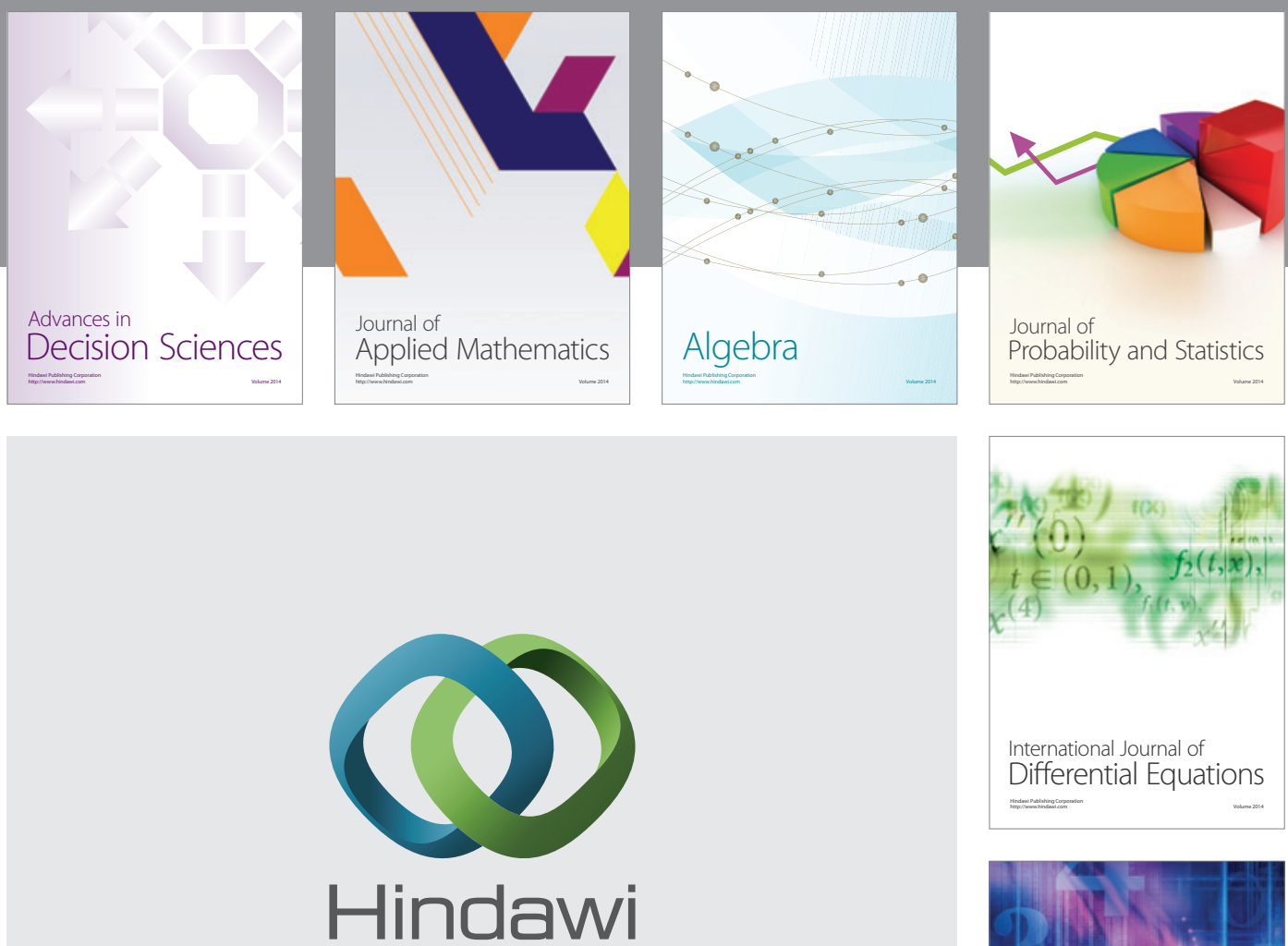

Submit your manuscripts at http://www.hindawi.com
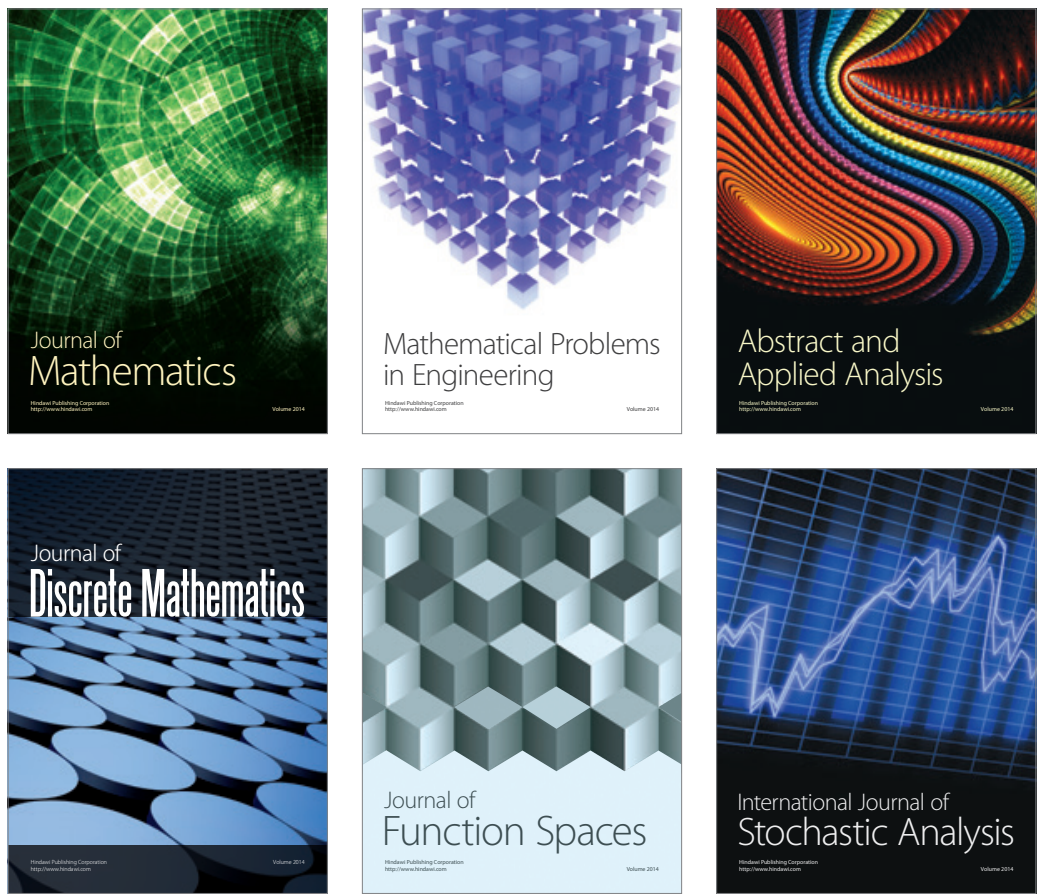

Journal of

Function Spaces

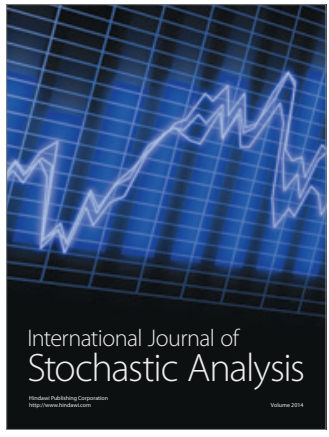

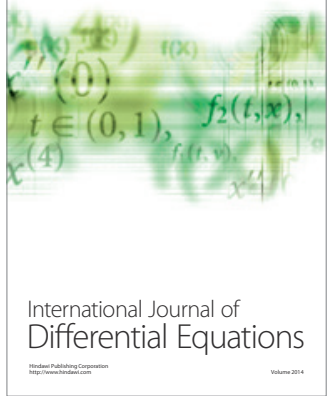
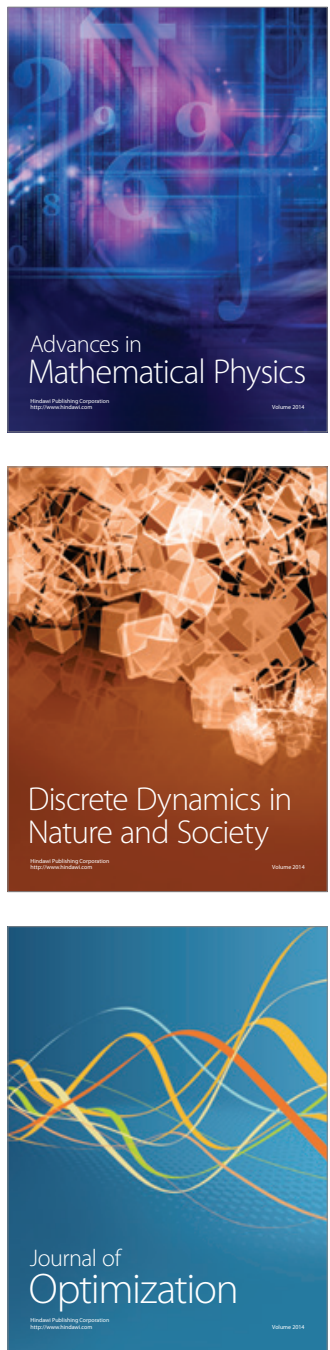